\title{
Evaluation of Maxillary Dental Arch Widths in Individuals Having Unilateral and Bilateral Cleft Lip and Palate Using Three-Dimensional Digital Models
}

\author{
Ege Dogan' ${ }^{1}$ Abdülkadir Işik², Servet Dogan³ \\ ${ }^{1}$ Private Office, Izmir, Turkey \\ ${ }^{2}$ İzmir Atatürk Eğitim ve Araştırma Hastanesi, Izmir, Turkey \\ ${ }^{3}$ Department of Orthodontics, Faculty of Dentistry, Ege University, Izmir, Turkey \\ Email: dtegedogan@hotmail.com, ikadir@msn.com, servetdgn@hotmail.com
}

How to cite this paper: Dogan, E., Işik, A. and Dogan, S. (2019) Evaluation of Maxillary Dental Arch Widths in Individuals Having Unilateral and Bilateral Cleft Lip and Palate Using Three-Dimensional Digital Models. Open Journal of Stomatology, 9, 159-167.

https://doi.org/10.4236/ojst.2019.97016

Received: May 22, 2019

Accepted: July 6, 2019

Published: July 9, 2019

Copyright $\odot 2019$ by author(s) and Scientific Research Publishing Inc. This work is licensed under the Creative Commons Attribution International License (CC BY 4.0).

http://creativecommons.org/licenses/by/4.0/

c)

\begin{abstract}
Aim: Maxillary dental arch widths were evaluated in individuals having unilateral (UCLP) and bilateral (BCLP) cleft lip and palate (CLP) using three-dimensional (3D) digital models. Material and Method: The study had been conducted on 80 individuals aged between $14-17$ years having UCLP and BCLP. 40 of the individuals had UCLP, whereas 40 had BCLP. The maxillary dental models taken from patients before the treatment were scanned using Orthomodel Programme (v.1.01, Orthomodel Inc., Istanbul, Turkey) to obtain 3D imagery. Student's t-test was used in order to assess the data obtained by using SPSS software version 22.0. Results: In BCLP, the average inter-canine distance was $17.44 \pm 1.31 \mathrm{~mm}$, the average inter-molar distance was $36.57 \pm 1.12 \mathrm{~mm}$, while inter-canine/inter-molar ratio was 0.47 . Whereas in UCLP, it was $25.10 \pm 0.63 \mathrm{~mm}, 42.20 \pm 0.53 \mathrm{~mm}$ and 0.59 . The inter-canine distance in UCLP was found to be large enough to be statistically significant $(p<0.01)$, whereas inter-molar distance was found not to be statistically ( $p>$ $0.05)$, even though there were differences in inter-molar widths. Conclusion: For the stable orthodontic treatment results, one of the most important points is arch form and widths to be coherent with each other. In our study, the increase of inter-canine distance seen in UCLP indicates that in the cleft region, the maxillary arch is inclined over to the back, while the same situation in BCLP suggests that the maxillary segments are collapsed inside. The difference in the arch is highly affected by the primary surgical treatment.
\end{abstract}

\section{Keywords}

Unilateral Cleft Lip and Palate, Bilateral Cleft Lip and Palate, 
Three-Dimensional Digital Models

\section{Introduction}

The patients with cleft lip and palate (CLP) are associated with certain anatomical defects. The lip, nose and alveolus have a cleft at the right or left side or bilateral cleft on each side. The cleft then continues into the palatal part of the maxilla and separates the palatal bone at the level of the nasal septum. In unilateral clefts (UCLP), the alveolar arch and palate are separated into a large and a small segments. In bilateral clefts (BCLP), the alveolar arch and palate are separated into two small segments. Maxillary arch dimensions are generally reduced in patients with CLP and are more reduced with complete clefts than with incomplete clefts. The primary surgical repairs affect the maxillary arch dimensions in patients with CLP. Lip repair in CLP has a moulding effect on the forward and outward rotated segments, and the surgical closure of the palate in both UCLP and BCLP children affect the growth of the maxillary arch in transverse and the antero-posterior dimensions [1]-[7].

With the help of Goslon Yardstick and the 5-Year-Old Index, maxillary arch constriction and occlusion can be evaluated from dental models in patients with CLP. However, as they are exclusively made for scoring patients with UCLP, a calibration course is needed to use the indices [4] [5]. Another crossbite scoring method is developed by Huddart and Bodenham to evaluate the maxillary arch constriction in patients with CLP [8]. It is more reliable to interarch discrepancies and correlate with the 5-year-old and Goslon indices [4] [5] [7] [8]. This method can be used on the cast or on the digital photographs of study models in BCLP and UCLP patients [8].

Impressions of the dental arches give a way to evaluate and compare the results of patients with CLP. From the impressions, dental plaster casts are made and these are used for measurements where parameters are performed and used to compare the patients having different types of clefts. 3D digital models are being used much more in recent years and they are alternative to conventional measurements on plaster models. High degree of validity is achieved by digital models, which are clinically acceptable. Linear and angular measurements which describe the arch form and palatal morphology, can be assessed. More recently, several studies used different three-dimensional (3D) imaging systems to accurately record the upper arch and palate in cleft subjects [8] [9] [10] [11] [12].

The aim of this study was to evaluate and compare arch widths using $3 \mathrm{D}$ digital study models in individuals with UCLP and BCLP.

\section{Material and Method}

The study has been conducted on 80 individuals aged between 14 - 17 years (40 male, 40 female) each having BCLP and UCLP primary and secondary CLP who 
referred to Ege University, Faculty of Dentistry Department of Orthodontics. 40 of the patients had BCLP, whereas 40 of them (18 right, 22 left) had UCLP. The maxillary dental models taken from patients were scanned with an orthodontic 3D scanner and the inter-canine and inter-molar widths and the ratio between these measurements were done by using Orthomodel Programme (v.1.01, Orthomodel Inc., Istanbul, Turkey).

The inclusion criteria were as follows:

Patients with UCLP and BCLP aged between 14 - 17 years.

The regular and unbroken maxillary dental models taken from patients before orthodontic treatment were chosen.

The exclusion criteria were as follows:

Patients with UCCLP and BCLP not aged between 14 - 17 years.

The irregular and broken maxillary dental models taken from patients before orthodontic treatment were not included to this study.

All the patients had orthodontic treatment later.

The measurements used in this study to evaluate maxillary dental arch widths in BCLP and UCLP patients were (Figure 1):

Inter-canine distance $(\mathrm{mm})$ : the distance between the tip of the canine tooth in the right and left segments in the maxillary segment.

Inter-molar distance $(\mathrm{mm})$ : the distance between the median point of palatinal and buccal tubercle of first molar tooth in the right and left segments in maxillary segment.

The number of the patients to be included in the study was determined by power analysis carried out by using the G Power analysis software. Student $t$ test was utilized in order to assess the data obtained. Statistical significance was set at $\mathrm{p}<0.05$.

The principles outlined in the Declaration of Helsinki were followed. Informed consents were signed by the parents of all the patients.

\section{Results}

Power analysis was performed by the computer program $\mathrm{G}$ power to determine and all sample sizes were required to achieve a representation rate of over $80 \%$. All measurements were repeated by the same observer over 10 randomly selected dental casts at least two weeks later for intra-observer reliability.

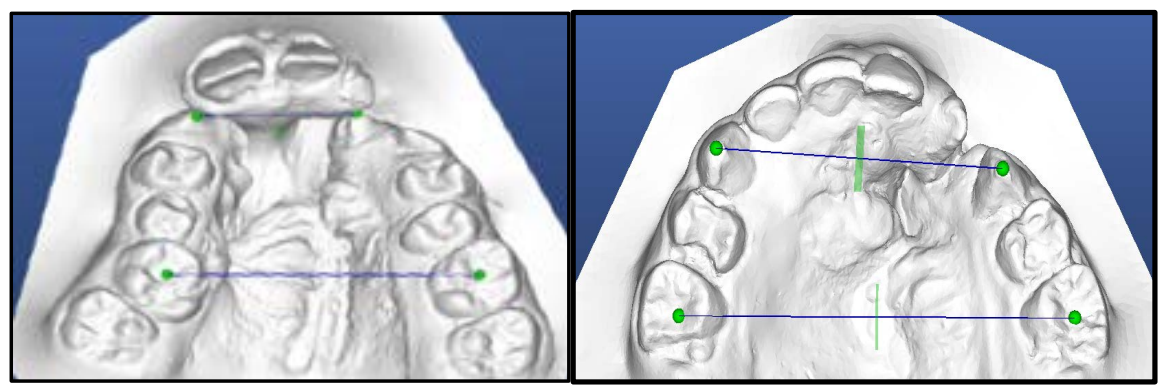

Figure 1. 3D digital models in UCLP and BCLP. 
In 40 individuals having BCLP aged between 14 - 17 years, the average inter-canine distance was $17.44 \pm 1.31 \mathrm{~mm}$, the average inter-molar distance was $36.57 \pm 1.12 \mathrm{~mm}$, while inter-canine/inter-molar ratio was 0.47 . Whereas in 40 individuals having UCLP (18 right, 22 left), the average inter-canine distance was found to be $25.10 \pm 0.63 \mathrm{~mm}$., the average inter-molar distance was $42.20 \pm$ $0.53 \mathrm{~mm}$, while inter-canine/inter-molar ratio was 0.59 . The inter-canine distance in UCLP were found to be large enough to be statistically significant ( $\mathrm{p}<$ $0.01)$, whereas inter-molar width were found not to be statistically ( $p>0.05$ ), even though there were differences in inter-molar widths. Besides, there was no statistically difference in inter-canine/inter-molar ratio in the two groups ( $p>$ 0.05) (Table 1, Figure 2).

\section{Discussion}

It has to be considered that in CLP patients, narrow upper arch is mostly seen which is a result of surgical treatment. Also, it is found out that untreated adults with CLP have reduced upper widths [1] [2] [3] [4]. This shows us that the reason of the constriction of the upper arch is not only caused by early surgical procedures but also caused by deficient palatal growth resulted because of the pressure of the surrounding tissues and maxillary segments and space between the palatal halves. Anterior part of the dental arch of patients with CLP is affected by the lip repair surgery, which causes a restrictive shaping effect. Orthopedically

Table 1. Maxillary dental arch widths in UCLP and BCLP $\left(\mathrm{p}<0.01^{\star *}\right)$

\begin{tabular}{|c|c|c|c|c|c|c|}
\hline & $\begin{array}{c}\text { inter-canine } \\
\text { distance (mm) }\end{array}$ & \multirow[t]{2}{*}{$p$ value } & $\begin{array}{c}\text { inter-molar } \\
\text { distance }(\mathrm{mm})\end{array}$ & \multirow[t]{2}{*}{$p$ value } & \multirow{2}{*}{$\begin{array}{c}\text { inter-canine/ } \\
\text { inter-molar ratio }\end{array}$} & \multirow[t]{2}{*}{ p value } \\
\hline & $\mathbf{x} \quad$ SD & & $\mathbf{x} \quad$ SD & & & \\
\hline $\begin{array}{l}\text { UCLP } \\
\mathrm{N}: 23\end{array}$ & $25.10 \pm 0.63$ & $\begin{array}{c}0.002 \\
* *\end{array}$ & $42.20 \pm 0.53$ & $\begin{array}{l}0.332 \\
\text { NS }\end{array}$ & 0.59 & $\begin{array}{l}0.523 \\
\text { NS }\end{array}$ \\
\hline $\begin{array}{l}\text { BCLP } \\
\mathrm{N}: 27\end{array}$ & $17.44 \pm 1.31$ & & $36.57 \pm 1.12$ & \multicolumn{3}{|c|}{0.47} \\
\hline
\end{tabular}

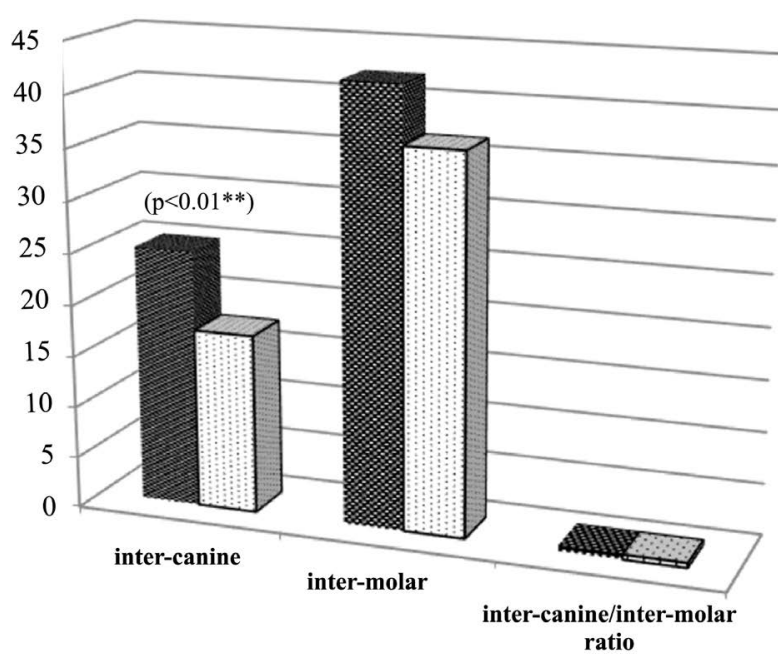

UCLP

unilateral clelf lip and palate

BCLP

bilateral clelf lip and palate

Figure 2. The comparison of maxillary dental arch widths in UCLP and BCLP $\left(\mathrm{p}<0.01^{\star *}\right)$. 
or orthodontically untreated patients who are in the mixed dentition having CLP show different upper arch morphology with maxillary constriction [9]-[16].

As the result of the reduced transverse maxillary arch widths, crossbite is an early and common malocclusion in children with CLP. For UCLP patients, investigators reported that anterior crossbite ranges from $7 \%$ to $64 \%$ and posterior crossbite ranges from $30 \%$ to $97 \%$. But in only cleft palate patients, the reported frequencies are lower, ranging from $14 \%$ to $27 \%$ for anterior crossbite and $22 \%$ to $37 \%$ for posterior crossbite. Subsequent to the presence of the cleft, reduction in the maxillary interdental width and tooth width is generally observed. Considering the requirement of exact evaluation of tooth size for achieving an esthetic, stable, and functional occlusal relationship, awareness about the variations in tooth size in patients with CLP may guide clinicians in orthodontics and accompanying dental treatment planning [15] [16] [17] [18].

In CLP research, a common approach for studying the effects of different treatment protocols is through evaluation of maxillary arch dimensions and occlusion from dental casts. Different surgical treatment protocols are frequently compared through the outcome of maxillary arch dimensions and occlusion. The studies about the measurements of the dental arch are key to define the most appropriate treatment planning for the cleft type and the cleft severity. The documentation protocol enables to evaluate the dental arch growth changes at a long term [9] [12] [15] [18].

The upper arch morphology of CLP patients are examined mainly by using conventional two dimensional (2D) dental casts analysis measured by directly and conventionally with digital calipers, which is a time consuming method with a limitation in providing reliable volumetric data. Some studies used 3D analysis for evaluating the upper ach dimensions in patients with CLP and comparison of arch widths were generally performed between untreated CLP patients and controls who were in the deciduous dentition [14] [16] [18].

Studies have showed that the patients having complete UCLP and BCLP have more altered inter-canine distance than the control group with a statistically significant smaller value. This could be the reason of the loss in the continuity of the alveolar ridge and supra position of the lateral segments on the cleft side. Patients with cleft palate have a close inter-canine distance when compared to non-cleft patients [9] [12] [15] [19].

Lione et al. [18], examined the variations of palatal morphology and maxillary arch size in patients who had prolonged mouth breathing caused by allergic rhinitis and compared to control group with normal breathing by using 3D analysis on digital casts. Patients with mouth breathing patterns showed an increase in palatal height and construction in the maxillary arch when compared to normal breathing patients.

Mello et al. [19], compared the anterior transverse dimensions of the dental arch of newborns by means of 3D digital casts with and without CLP. UCLP group had mean of $36.5 \mathrm{~mm}$, BCLP group had a mean of 34.8 and control group had a mean of $27.52 \mathrm{~mm}$. Statistically significant difference was found between 
the control group and both CLP groups. No statistically significant difference between complete UCLP and BCLP groups were found. They observed that inter-canine distance was greater in UCLP patients than in complete BCLP patients and those without CLP.

Fernandes et al. [20], compared the dental arch dimensions on 3D digital study models in patients with and without CLP before the primary surgery. They examined the 223 digital models of patients aged between 3 - 9 months. The results showed that the intercanine distance and anterior cleft widths were wider in patients with UCLP. The intertuberosity distances and posterior cleft width were wider in patients having BCLP among the groups. Patients with CLP had wider maxillary arch dimensions than the children without CLP before the primary surgery.

Heidbuchel, K.L. et al. [21], described the growth of maxillary arch dimensions in patients with BCLP until the age of 4 and compared them to non-cleft patients. The comparison between these two groups was made at fixed time intervals. At birth, anterior and posterior arch widths and arch depths were significantly larger in patients having BCLP. At the $7^{\text {th }}$ month after the lip closure, anterior arch width and arch depth diminished considerably in the cleft group. At the 12th month after palatoplasty, a slight decrease in posterior arch width was seen. At the age of 4 , anterior arch width was significantly narrower and anterior arch depth was shorter in patients having BCLP. Posterior arch width was significantly wider. Maxillary arch dimensions in patients with BCLP show a unique development that is significantly different from that in noncleft patients during the first 4 years of life.

Smahel et al. [22], reported reduced dentoalveolar widths in isolated palatal clefts in the upper arch, but the reduction increased in the posterior direction compared to controls. Arch was wider in the anterior part and narrow in the posterior part compared to UCLP, this may be the reason of the less compression of the intact anterior part of the arch. On the other hand, a comparison between BCLP and UCLP showed an almost double narrowing of the upper arch at the level of the canines in BCLP, although between molars, the difference was less evident.

Maxillary arch dimensions are generally reduced in patients with clefts, and are more reduced with complete clefts than with incomplete clefts. The primary surgical repairs affect maxillary arch dimensions in children with clefts. Lip repair in CLP has a moulding effect on the forward and outward rotated segments, which creates a more normal alveolar arch shape and the surgical closure of the palate in CLP affects the growth of the maxillary arch in transverse and antero-posterior dimensions. The dimensions of maxillary dental arches and measurements of cleft width play an important role in deciding the treatment plan for each type of CLP. Accordingly, expansion of the anterior part of the maxillary arch could be beneficial [1] [2] [3] [4] [10] [15] [21]. In our study, the inter-canine distance in UCLP were found to be large enough to be statistically significant $(\mathrm{p}<0.01)$, whereas inter-molar widths were found not to be statisti- 
cally ( $p>0.05$ ), even though there were differences in inter-molar widths. Orthopedic expansion of patients with CLP, with a stress in the anterior section of the palate, would be beneficial in order to provide needed space for the tongue and allowing for normal growth and development.

\section{Conclusions}

The increase of inter-canine distance seen in UCLP indicates that in the cleft region, the maxillary arch is inclined over to the back, while the same situation in BCLP suggests that the maxillary segments are collapsed inside. The difference in the arch is actively affected by the primary surgical treatment. Therefore, CLP patients show a significantly different upper arch morphology, with a higher degree of maxillary constriction. In order to achieve stable orthodontic treatment, one of the most important points is to make the correct treatment planning for the arch forms and widths.

3D evaluations of the maxillary arch highlight significant differences between individuals having UCLP and BCLP who are in mixed dentition period, where orthopedic maxillary expansion is advisable in both groups.

\section{Conflicts of Interest}

The authors declare no conflicts of interest regarding the publication of this paper.

\section{References}

[1] Latief, B.S. Lekkas, C. and Kuijpers Jagtman, A.M. (2009) Maxillary Arch Width in Unoperated Adult of the Unilateral Cleft Lip and Alveolus Patients. Journal of Maxillofacial and Oral Surgery, 8, 218-220. https://doi.org/10.1007/s12663-009-0053-4

[2] Reiser, E., Skoog, V. and Andlin-Sobocki, A. (2013) Early Dimensional Changes in Maxillary Cleft Size and Arch Dimensions of Children with Cleft Lip and Palate and Cleft Palate. The Cleft Palate-Craniofacial Journal, 50, 481-490. https://doi.org/10.1597/11-003

[3] Liao, Y.F. and Mars, M. (2005) Long-Term Effects of Palate Repair on Craniofacial Morphology in Patients with Unilateral Cleft Lip and Palate. The Cleft Palate-Craniofacial Journal, 42, 594-600. https://doi.org/10.1597/04-077r.1

[4] Nollet, P.J., Katsaros, C., Van't Hof, M.A. and Kuijpers-Jagtman, A.M. (2005) Treatment Outcome in Unilateral Cleft Lip and Palate Evaluated with the GOSLON Yardstick: A Meta-Analysis of 1236 Patients. Plastic and Reconstructive Surgery, 116, 1255-1262. https://doi.org/10.1097/01.prs.0000181652.84855.a3

[5] Mars, M., Asher-McDade, C., Brattstrom, V., Dahl, E., McWilliam, J., Molsted, K., et al. (1992) A Six-Center International Study of Treatment Outcome in Patients with Clefts of the Lip and Palate: Part 3. Dental Arch Relationships. The Cleft Palate-Craniofacial Journal, 29, 405-408. https://doi.org/10.1597/1545-1569_1992_029_0405_asciso_2.3.co_2

[6] Nyström, M. and Ranta, R. (1989) Sizes of Dental Arches and Interdental Space in 3-Year-Old Children with and without Cleft Lip/Palate. European Journal of Orthodontics, 11, 82-88. https://doi.org/10.1093/oxfordjournals.ejo.a035969

[7] Mars, M., Plint, D.A. Houston, W.J., Bergland, O. and Semb, G. (1987) The Goslon 
Yardstick: A New System of Assessing Dental Arch Relationships in Children with Unilateral Clefts of the Lip and Palate. The Cleft Palate-Craniofacial Journal, 24, 314-322.

[8] Huddart, A.G. and Bodenham, R.S. (1972) The Evaluation of Arch form and Occlusion in Unilateral Cleft Palate Subjects. The Cleft Palate-Craniofacial Journal, 9 , 194-209.

[9] Fleming, P.S. Marinho, V. and Johal, A. (2011) Orthodontic Measurements on Digital Study Models Compared with Plaster Models: A Systematic Review. Orthodontics \& Craniofacial Research, 14, 1-16. https://doi.org/10.1111/j.1601-6343.2010.01503.x

[10] Bejdovà, S., Krajìcek, V., Peterka, M., Trefny, P. and Velemìnska, J. (2012) Variability in Palatal Shape Size in Patients with Bilateral Complete Cleft Lip and Palate Assessed Using Dense Surface Model Construction and 3D Geometric Morphometrics. Journal of Cranio-Maxillofacial Surgery, 40, 201-208. https://doi.org/10.1016/j.jcms.2011.04.013

[11] Primozic, J., Baccetti, T., Franchi, L., Richmond, S., Farcnik, F. and Ovsenik, M. (2011) Three-Dimensional Assessment of Palatal Change in a Controlled Study of Unilateral Posterior Crossbite Correction in the Primary Dentition. European Journal of Orthodontics, 35, 199-204. https://doi.org/10.1093/ejo/cjr125

[12] Ruskovà, H., Bejdovà, S., Peterka, M., Krajìcek, V. and Velemìnskà, J. (2013) 3-D Shape Analysis of Palatal Surface in Patients with Unilateral Complete Cleft Lip and Palate. Journal of Cranio-Maxillofacial Surgery, 42, 140-147. https://doi.org/10.1016/j.jcms.2013.07.011

[13] Botticelli, S., Pedersen, T.K., Küseler, A., Nørholt, S.E. and Cattaneo, P.M. (2019) Novel 3-D Analysis for the Assessment of Cleft Dimensions on Digital Models of Infants With Unilateral Cleft Lip and Palate. The Cleft Palate-Craniofacial Journal, 56, 127-133. https://doi.org/10.1177/1055665618770795

[14] Generali, C., Primozic, J., Richmond, S., Bizzarro, M., Flores-Mir, C., Ovsenik, M. and Perillo, L. (2017) Three-Dimensional Evaluation of the Maxillary Arch and Palate in Unilateral Cleft Lip and Palate Subjects Using Digital Dental Casts. European Journal of Orthodontics, 39, 641-645. https://doi.org/10.1093/ejo/cjx019

[15] Huang, C.S., Wang, W.I., Liou, E.J., Chen, Y.R., Chen, P.K., et al. (2002) Effects of Cheiloplasty on Maxillary Dental Arch Development in Infants with Unilateral Complete Cleft Lip and Palate. The Cleft Palate-Craniofacial Journal, 39, 513-516. https://doi.org/10.1597/1545-1569(2002)039<0513:EOCOMD>2.0.CO;2

[16] Mazaheri, M., Athanasiou, A.E., Long, R.E. and Kolokitha, O.G. (1993) Evaluation of Maxillary Dental Arch Form in Unilateral Clefts of Lip, Alveolus, and Palate from One Month to Four Years. The Cleft Palate-Craniofacial Journal, 30, 90-93. https://doi.org/10.1597/1545-1569_1993_030_0090_eomdaf_2.3.co_2

[17] Falzoni, M.M.M., Jorge, P.K., Laskos, K.V., Carrara, C.F.C., Machado, M.A.A., Valarelli, F.P. and Oliveira, T.M. (2016) Three-Dimensional Dental Arch Evaluation of Children with Unilateral Complete Cleft Lip and Palate. Dental, Oral and Craniofacial Research, 2, 238-241. https://doi.org/10.15761/DOCR.1000154

[18] Lione, R., Buongiorno, M., Franchi, L. and Cozza, P. (2013) Evaluation of Maxillary Arch Dimensions and Palatal Morphology in Mouth-Breathing Children by Using Digital Dental Casts. International Journal of Pediatric Otorhinolaryngology, 78, 91-95. https://doi.org/10.1016/j.ijporl.2013.09.028

[19] Mello, B.Z.F., Fernandes, V.M., Carrara, C.F.C., Machado, M.A.A.M. Garıb, D.G. and Olıveıra, T.M. (2013) Evaluation of the Intercanine Distance in Newborns with 
Cleft Lip and Palate Using 3D Digital Casts. Journal of Applied Oral Science, 21, 437-442. https://doi.org/10.1590/1679-775720130091

[20] Fernandes, V.M., Jorge, P.K., Carrara, C.F.C., Gomide, M.R., Machado, M.A.A.M. and Oliveira, T.M. (2015) Three-Dimensional Digital Evaluation of Dental Arches in Infants with Cleft Lip and/or Palate. Brazilian Dental Journal, 26, 297-302. https://doi.org/10.1590/0103-6440201300161

[21] Heidbuchel, K.L., Kuijpers-Jagtman, A.M., Kramer, G.J. and Prahl-Andersen, B. (1998) Maxillary Arch Dimensions in Bilateral Cleft Lip and Palate From Birth Until Four Years of Age in Boys. The Cleft Palate-Craniofacial Journal, 35, 233-239. https://doi.org/10.1597/1545-1569(1998)035<0233:MADIBC>2.3.CO;2

[22] Smahel, Z., Veleminska, J., Trefny, P. and Mullerova, Z. (2009) Three-Dimensional Morphology of the Palate in Patients with Bilateral Complete Cleft Lip and Palate at the Stage of Permanent Dentition. The Cleft Palate-Craniofacial Journal, 46, 399-408. https://doi.org/10.1597/07-199.1 